\title{
COMPRESSION OF THE DEEP PALMAR BRANCH OF THE ULNAR NERVE BY AN ANOMALOUS MUSCLE
}

\author{
Case Report and Review
}

\author{
A. K. Jeffery, Dunedin, New Zealand \\ From the Orthopaedic Department, Dunedin Public Hospital, \\ and the Department of Surgery, University of Otago Medical School, Dunedin
}

The deep branch of the ulnar nerve as it crosses the hand supplies the interossei, third and fourth lumbricals, the adductor pollicis and part of flexor pollicis brevis. Although variations in this distribution occur (Highet 1943, Rowntree 1949), a lesion of the nerve characteristically presents as wasting and weakness of these muscles with sparing of hypothenar muscles and normal sensibility. This pattern of paralysis is a well documented entity first described by Hunt (1908), who ascribed it to traumatic neuritis of the deep branch, related to the patient's occupation. Later reports appeared to support this hypothesis (Harris 1929, Worster-Drought 1929, Russell and Whitty 1947, Bakke and Wolff 1948).

Compression of the deep branch by a carpal ganglion was reported by Seddon (1952), who described four cases confirmed at operation. Since then, ganglia in this region have been recognised as a relatively frequent cause of compression either of the ulnar nerve itself or of its deep branch (Shea and McClain 1969). Whether or not a ganglion is present, many patients with this paralysis give a history of repeated trauma to the hand (Seddon 1952, Brooks 1963).

The following report describes a case in which compression of the deep branch was caused by an accessory abductor minimi digiti muscle. The abnormal muscle was hypertrophied, probably as a result of the patient's manual occupation. Five cases of ulnar nerve compression at the wrist by an anomalous muscle have previously been recorded. A review of these cases is presented, and the morphology and clinical presentation of anomalous muscles in this region are discussed.

\section{CASE REPORT}

A carpenter, fifty-six years old and right handed, was referred with a two years' history of increasing weakness in the right hand. He had first noticed the weakness when hammering nails into a ceiling above his head. He found it difficult to grip the hammer adequately; later he noticed clumsiness when manipulating screws and bolts. About this time he suffered a brief episode of burning pain in the hand, localised to the ring and little fingers and occurring at night when in bed. Over the succeeding months muscle wasting and clawing of the fingers became obvious. In the four weeks before he was seen the patient noticed a swelling on the ulnar border of the wrist. This was soft, non-tender, and appeared to fluctuate in size, being larger after work that involved hammering.

Examination revealed wasting of the interossei and adductor pollicis with clawing of the fingers (Fig. 1). There was marked weakness of all muscles commonly supplied by the deep branch of the ulnar nerve, and the patient could not abduct the index finger. The hypothenar muscles appeared normal in bulk and power, and the little finger was held in a slightly abducted position (Fig. 2). Sensibility to pinprick and light touch was normal. Examination of the wrist revealed a soft, ill-defined swelling on the volar surface, extending proximally into the forearm for about five centimetres, in the line of the flexor carpi ulnaris. A diagnosis was made of compression of the deep branch of the ulnar nerve by a ganglion. 
Operation and progress-A curved incision was made in the palm, parallel to and on the medial side of the thenar crease. The incision was continued along the flexion crease of the wrist towards the pisiform bone, then extended proximally into the forearm in a curved fashion.

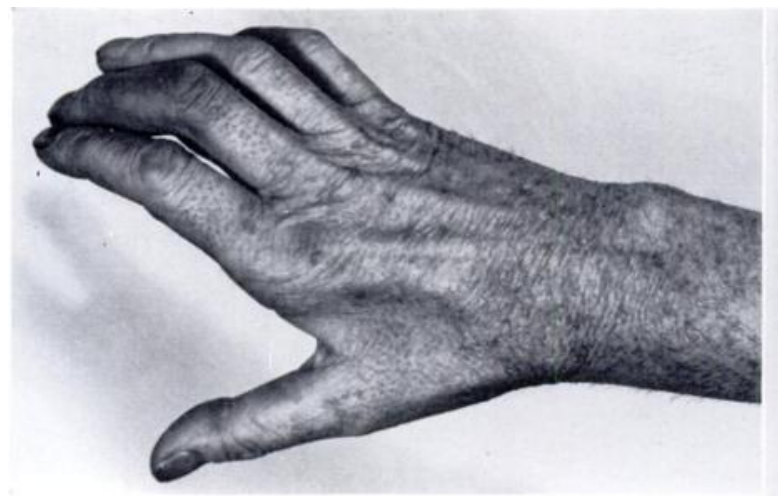

FIG. 1

Figs. 1 AND 2

Figure 1-The patient's hand showing wasting of the interossei and clawing of the fingers. Figure $2-$ The palm of the hand shows wasting of adductor pollicis with preservation of the hypothenar muscles. The little finger is held slightly abducted and an ill-defined swelling is present on the ulnar border of the wrist.

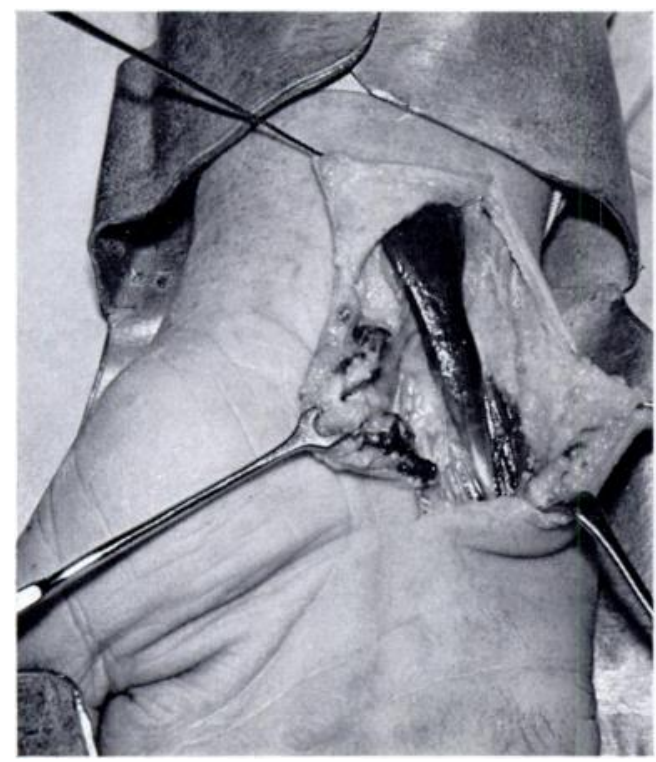

FIG. 3

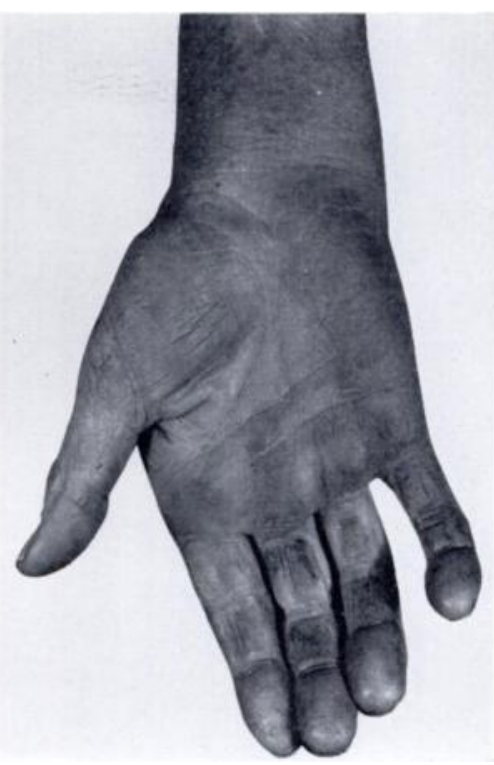

FIG. 2

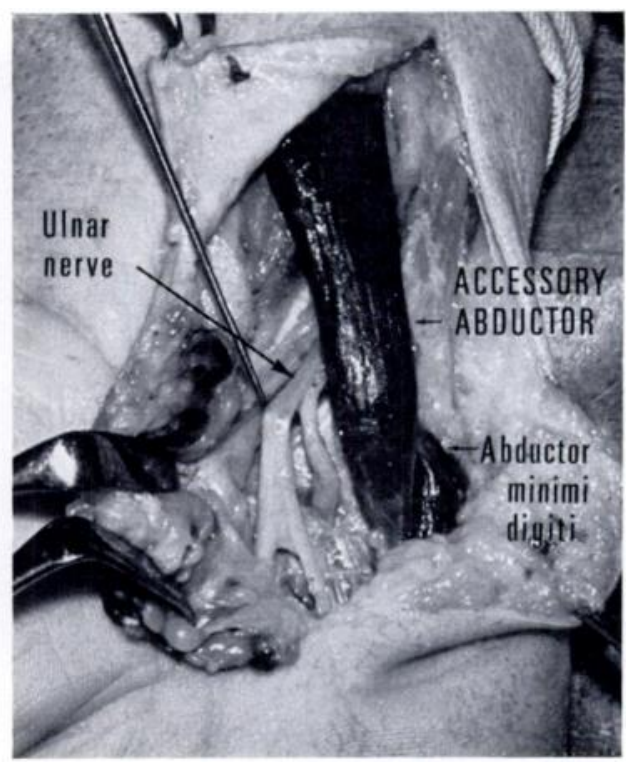

Fig. 4

Figure 3-The hypertrophied accessory abductor minimi digiti muscle found at operation. Palmaris brevis is retracted radially. Figure 4--The ulnar nerve retracted from beneath the accessory muscle. The deep branch of the nerve is thickened as it passes over the distal edge of the piso-hamate ligament.

It was immediately obvious that the swelling noted before operation was an anomalous muscle arising in the lower forearm and passing distally beneath the palmaris brevis muscle (Fig. 3). The muscle was dark red, and appeared hypertrophied. The palmaris longus muscle 
was absent. Palmaris brevis was reflected radially, with preservation of its nerve supply, and the abnormal muscle was defined.

The muscle arose from the deep fascia covering the flexor muscles in the lower forearm and received its nerve supply from the ulnar nerve just proximal to the pisiform bone. It passed distally between the pisiform and the hook of the hamate, and terminated as a narrow tendon fusing with the muscle belly of abductor minimi digiti on the ulnar side of the fifth metacarpo-phalangeal joint (Fig. 5).

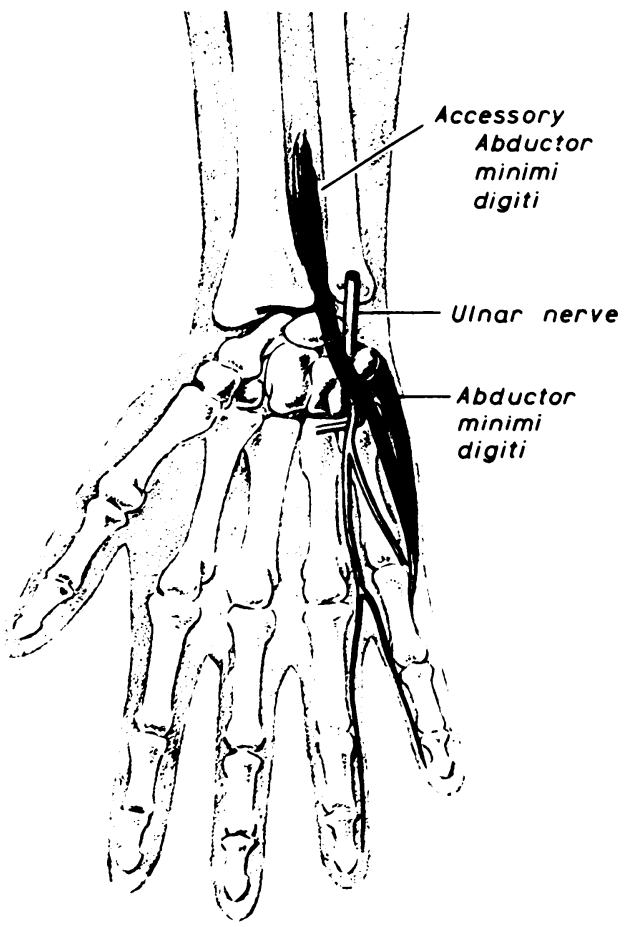

Fig. 5

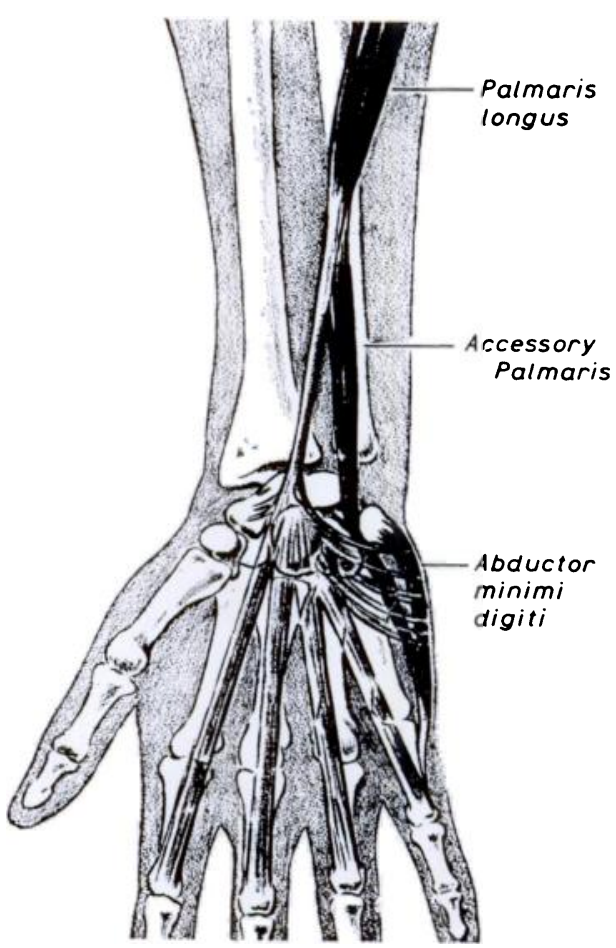

Fig. 6

Diagrams showing two anomalous muscles that may cause compression of the ulnar nerve at the wrist. Figure 5-The accessory abductor minimi digiti as noted at operation, showing its relationship to the ulnar nerve in the canal of Guyon. Palmaris longus is absent. Figure 6-The accessory palmaris. Although arising from the palmaris longus, this muscle appears more closely related to the abductor minimi digiti, into which it is commonly inserted.

By retracting the abnormal muscle medially the ulnar nerve was defined. The deep branch was thickened as it passed over the distal edge of the piso-hamate ligament (Fig. 4). Exploration of this branch into the palm of the hand failed to show any other pathology. The muscle was excised: it measured ten centimetres in length.

The patient returned to his work as a carpenter. When seen nine months later he claimed that there had been a marked improvement in the hand, both in power and in ability to perform fine movements. The clawing was less obvious, and the patient could now abduct the index finger.

\section{MORPHOLOGY AND VARIATIONS OF HYPOTHENAR MUSCLES AND PALMARIS LONGUS}

Abductor minimi digiti-The abductor minimi digiti arises chiefly from the lower border and medial surface of the pisiform bone, and the ligaments between it and the hamate bone, and is inserted into the base of the first phalanx of the little finger on its ulnar side, a slip being sent to the extensor tendon (Quain 1923). 
Variations of this muscle are frequent and have been well described by anatomists, notably Le Double (1897) and Bryce (1923). The muscle may be absent or fused with flexor brevis minimi digiti. It is occasionally divided into two or three fasciculi (Le Double 1897).

An accessory head may be present and can arise from the tendon of flexor carpi ulnaris or from the flexor retinaculum; or it may arise a considerable distance above the wrist from the intermuscular fascia under either flexor carpi radialis or flexor carpi ulnaris (Bryce 1923). As seen in the present case report, an accessory head may also arise from the fascia of the forearm (Fig. 5). This particular anomaly was described by Smith (1895), who cited reports of four similar cases. Smith noted, when dissecting a cadaver, that the abductor minimi digiti of the right hand, in addition to its usual origin, had a broad fan-like origin from the deep fascia of the forearm about five centimetres above the wrist. The muscle extended downwards across the ulnar artery and nerve to the radial side of the pisiform bone, where it joined the other part of the muscle. As in the present case, the muscle was supplied by a twig from the ulnar nerve, and the palmaris longus muscle was absent.

Palmaris longus-Palmaris longus, like abductor minimi digiti, frequently shows variations in form. Phylogenetically, both of these muscles are similar in that they represent parts of the most superficial sheet of muscle in the developing forearm and hand (McMurrich 1902, 1903). Accessory muscles may arise from palmaris longus and insert into the hypothenar muscles. In a study of anomalies of the palmaris longus by Reimann, Daseler, Anson and Beaton (1944), accessory slips were found in fifteen out of 530 limbs studied. The commonest accessory slip was a small muscle arising from the palmaris longus tendon and inserting into the abductor minimi digiti belly (Fig. 6). In one case the muscle was noted to insert into flexor minimi digiti. Although named accessory palmaris, it has been pointed out by King and O'Rahilly (1950) that this muscle slip is usually supplied by the ulnar nerve, and appears more closely related to the hypothenar muscles than to palmaris longus. It therefore seems more correct to consider the accessory palmaris as an accessory belly of either the abductor minimi digiti or the flexor minimi digiti when it is inserted into one of these muscles.

Palmaris brevis, opponens and flexor minimi digiti-Variations in these hypothenar muscles are recorded, but they seem to occur less frequently. The opponens and flexor minimi digiti may be fused to each other or to abductor minimi digiti, or they may be absent (Le Double 1897). Palmaris brevis is rarely absent but varies greatly in the strength and direction of its fibres (Bryce 1923). Lipscomb (1960) recorded a case in which a large palmaris brevis muscle together with an anomalous three-headed muscle presented clinically as a mass in the hypothenar region, simulating a soft-tissue neoplasm.

\section{DISCUSSION}

Previous case reports-In addition to the present case in which the deep branch was involved, a further five cases have been described in which the ulnar nerve itself was compressed at the wrist by an anomalous muscle.

In Thomas's (1958) case compression was caused by an accessory palmaris muscle (Fig. 6). Although the exact point of insertion was not stated, the muscle arose from the tendon of palmaris longus and passed distally on the radial side of the pisiform bone to gain insertion in the region of the hypothenar muscles. The patient, a man aged twenty, presented with symptoms of fatigue and a "dead" feeling on use of the left hand. A mass was present on the volar aspect of the left wrist and the pre-operative diagnosis of a ganglion was made. Excision of the muscle resulted in relief of symptoms.

In a brief report by Muller (1963) three cases were described in which abnormal muscle tissue caused ulnar nerve compression at the wrist. Details of the muscle anomalies 
were not given, but it was noted that improvement was seen after removing the cause of compression.

Schjelderup (1964) reported the case of a thirty-four years old carpenter who had experienced increasing pain in the sensory area of the ulnar nerve in the right hand, with motor disturbance. Symptoms were worse during work. At operation an abnormal muscle was found crossing the ulnar nerve just before its division into sensory and motor components and compressing the nerve at this point. Details of the origin and insertion of the muscle were not given.

Anatomical features--The ulnar nerve enters the hand through the narrow confines of a canal bounded by the pisiform and hamate bones (canal of Guyon 1861). An accessory muscle arising in the distal forearm and gaining insertion into the hypothenar muscles also passes through the canal, lying superficial to the nerve (Figs. 5 and 6). In the case reported in this paper the findings at operation suggested that in this region the hypertrophied muscle was compressing the deep branch of the ulnar nerve against the piso-hamate ligament. Anatomical dissections by Hayes, Mulholland and O'Connor (1969) have shown that at the distal border of the piso-hamate ligament the deep branch passes beneath a ligamentous arch and is particularly vulnerable to compression at this point. The brief episode of paraesthesiae in the ring and little fingers suggests that pressure by the abnormal muscle may have also involved the main trunk of the nerve proximal to its division, although no evidence of this was found at operation.

Symptoms and their relationship to manual work-Although frequently seen in the dissection room, anomalous muscles in the hand or wrist seldom produce clinical symptoms. When symptoms do occur, they appear to be related to two factors: the anatomical site of the muscle and the presence of muscle hypertrophy. This is seen with an anomalous muscle, the extensor brevis digitorum manus, which may present as a painful swelling on the dorsum of the hand. In a review of its clinical presentation, Ross and Troy (1969) noted that the symptoms usually occurred in men and followed heavy manual work, and that the swelling was frequently diagnosed as a ganglion.

With the anomalous hypothenar muscle (including the accessory palmaris), symptoms of ulnar nerve compression may be the presenting feature, particularly when the accessory muscle belly arises in the distal forearm and passes with the ulnar nerve through the canal of Guyon. Like the anomalous extensor brevis digitorum manus, however, prolonged or repeated use of the hand may aggravate the symptoms by causing hypertrophy of the abnormal muscle. Of the three cases reviewed in this paper in which the relevant details were recorded, symptoms of ulnar nerve compression were related to manual work, two of the patients being carpenters. In the two cases in which a swelling was also noted, a pre-operative diagnosis of ganglion was made.

\section{SUMMARY AND CONCLUSIONS}

1. A case of compression of the deep palmar branch of the ulnar nerve by an accessory abductor minimi digiti muscle is described.

2. The morphology of abnormal muscles in the hypothenar region is discussed.

3. Five previously reported cases of ulnar nerve compression at the wrist by an anomalous muscle are reviewed.

4. When symptoms are produced by an anomalous hypothenar muscle, they seem to be related to the anatomical site of the muscle and the presence of muscle hypertrophy. Occupational factors may be important in producing this hypertrophy.

1 would like to thank $\mathrm{Dr}$ M. Pollock, who first saw this patient, Mr L. J. Blackman for the photographs, and $\mathrm{Mr}$ J. B. Irwin for the diagrams. 
REFERENCES

Bakke, J. L., and Wolff, H. G. (1948): Occupational Pressure Neuritis of the Deep Palmar Branch of the Ulnar Nerve. Archives of Neurology and Psychiatry, 60, 549.

Brooks, D. M. (1963): Nerve Compression Syndromes. Journal of Bone and Joint Surgery, 45-B, 445.

Bryce, T. H. (1923): In Quain's Elements of Anatomy. Eleventh edition, Vol. IV, Part II, Myology. London: Longmans, Green \& Co.

GuYon, F. (1861): Note sur une disposition anatomique propre à la face antérieure de la région du poignet et non encore décrite. Bulletins de la Société Anatomique de Paris, second series, 6, 184.

Harris, W. (1929): Occupational Pressure Neuritis of the Deep Palmar Branch of the Ulnar Nerve. British Medical Journal, 1, 98.

Hayes, J. R., Mulholland, R. C., and O’Connor, B. T. (1969): Compression of the Deep Palmar Branch of the Ulnar Nerve. Journal of Bone and Joint Surgery, 51-B, 469.

Highet, W. B. (1943): Innervation and Function of the Thenar Muscles. Lancet, i, 227.

Hunt, J. R. (1908): Occupation Neuritis of the Deep Palmar Branch of the Ulnar Nerve. Journal of Nervous and Mental Diseases, 35, 673.

KING, T. S., and O'Rahilly, R. (1950): M. Palmaris Accessorius and Duplication of M. Palmaris Longus. Acta Anatomica, 10, 327.

LipscomB, P. R. (1960): Duplication of Hypothenar Muscles Simulating Soft-tissue Tumor of the Hand. Journal of Bone and Joint Surgery, 42-A, 1058.

LF Double, A.-F. (1897): Traité des variations du système musculaire de l'homme, et de leur signification au point de vue de l'anthropologie zoologique. Vo. II. Paris: Schleicher Frères.

MCMurrich, J. P. (1902): The Phylogeny of the Forearm Flexors. American Journal of Anatomy, 2, 177.

MCMurrich, J. P. (1903): The Phylogeny of the Palmar Musculature. American Journal of Anatomy, 2, 463.

Muller, L. H. (1963): Anatomical Abnormalities at the Wrist Joint Causing Neurological Symptoms in the Hand. Journal of Bone and Joint Surgery, 45-B, 431.

Quain, J. (1923): Elements of Anatomy. Eleventh edition, Vol. IV, Part II, Myology. London: Longmans, Green \& Co.

Rfimann, A. F., Daseifer, E. H., Anson, B. J., and Beaton, L. E. (1944): The Palmaris Longus Muscle and Tendon. Anatomical Record, 89, 495.

Ross, J. A., and Troy, C. A. (1969): The Clinical Significance of the Extensor Digitorum Brevis Manus. Journal of Bone and Joint Surgery, 51-B, 473.

Rowntree, T. (1949): Anomalous Innervation of the Hand Muscles. Journal of Bone and Joint Surgery, 31-B, 505.

Russell, W. R., and Whitty, C. W. M. (1947): Traumatic Neuritis of the Deep Palmar Branch of the Ulnar Nerve. Lancet, $1,828$.

Schuelderup, H. (1964): Aberrant Muscle in the Hand Causing Ulnar Nerve Compression. Journal of Bone and Joint Surgery, 46-B, 361.

Stddon, H. J. (1952): Carpal Ganglion as a Cause of Paralysis of the Deep Branch of the Ulnar Nerve. Journal of Bone and Joint Surgery, 34-B, 386.

SheA, J. D., and McClain, E. J. (1969): Ulnar-nerve Compression Syndromes at and below the Wrist. Journal of Bone and Joint Surgery, 51-A, 1095.

Sмiтн, G. E. (1895): An Account of Some Rare Nerve and Muscle Anomalies, with Remarks on Their Significance. Journal of Anatomy and Physiology, 29, 84.

Thomas, C. G., Jun. (1958): Clinical Manifestations of an Accessory Palmaris Muscle. Journal of Bone and Joint Surgery, 40-A, 929.

Worster-Drought, C. (1929): Pressure Neuritis of the Deep Palmar Branch of the Ulnar Nerve. British Medical Journal, 1, 247.

VOL. 53 B, NO. 4, NOVEMBER 1971 